\title{
A Method for Calculating Hard QoS Guarantees for Networks-on-Chip
}

\author{
Dara Rahmati ${ }^{\star}$, Srinivasan Murali ${ }^{\sharp}$, Luca Benini ${ }^{\ddagger}$, Federico Angiolini ${ }^{\sharp}$, \\ Giovanni De Micheli ${ }^{\S}$, Hamid Sarbazi-Azad* \\ *HPCAN, Sharif University of Technology, Tehran, Iran, \{d_rahmati@ce.sharif.edu, azad@sharif.edu\} \\ *iNoCs Sarl, \{murali, angiolini\}@inocs.com \\ §LSI, EPFL, Lausanne, Switzerland, giovanni.demicheli@epfl.ch \\ ‡DEIS, University of Bologna, Bologna, Italy, luca.benini@unibo.it
}

\begin{abstract}
Many Networks-on-Chip (NoC) applications exhibit one or more critical traffic flows that require hard Quality of Service (QoS). Guaranteeing bandwidth and latency for such real time flows is crucial. In this paper, we present novel methods to efficiently calculate worst-case bandwidth and latency bounds and thereby provide hard QoS guarantees. Importantly, the proposed methods apply even to best-effort NoC architectures, with no extra hardware dedicated to QoS support. By applying our methods to several realistic NoC designs, we show substantial improvements (on average, more than $30 \%$ in bandwidth and $50 \%$ in latency) in bound tightness with respect to existing approaches. ${ }^{1}$
\end{abstract}

\section{Categories and Subject Descriptors}

B.4.3 [Hardware]: Interconnections (Subsystems)

C.1.2 [Processor Architectures]: Multiple Data Streaming Architecture (MultiProcessor) - Interconnection Architectures

\section{General Terms}

Algorithms, Performance and Design.

\section{Keywords}

SoC, NoC, QoS, Wormhole switching, Real-time guarantees, Performance analytical model.

\section{INTRODUCTION}

The Networks-on-Chip [1,2] paradigm has emerged in recent years to overcome the scalability limitations of point-to-point signal wires, shared buses or segmented buses [1,3], which do not scale well in power, performance and design complexity [4, $5,6]$. While the scalability and efficiency advantages of NoCs have been demonstrated in many occasions, their timing predictability and suitability to transport real-time (RT) communication are still a source of technical concern.

Many applications have strict requirements on latency and bandwidth of on-chip communication, which are often expressed as real-time constraints on inter-core traffic flows.

Permission to make digital or hard copies of all or part of this work for personal or classroom use is granted without fee provided that copies are not made or distributed for profit or commercial advantage and that copies bear this notice and the full citation on the first page. To copy otherwise, or republish, to post on servers or to redistribute to lists, requires prior specific permission and/or a fee.

ICCAD'09, November 2-5, 2009, San Jose, CA, USA.

Copyright 2009 ACM 978-1-60558-800-1/09/11...\$10.00
On a NoC fabric this translates to guaranteed QoS requirements for packet delivery. Different approaches have been used to support guaranteed QoS for NoCs: priority-based switching schemes [7], time-triggered communication [8], time-division multiple-access [9] and many variations of these ideas. All these approaches imply hardware overhead and often come with strict service disciplines that limit NoC flexibility and penalize average performance to provide worst-case guarantees. In fact, NoCs prototypes are often classified as being either best-effort or QoS, depending on the availability of hardware support for RT traffic.

Our work takes a new viewpoint. We consider best-effort NoC architectures without special HW support for QoS traffic. We only assume that the traffic injected by network end-nodes is known and characterized in terms of its worst-case behavior. We then formulate algorithms to find conservative latency and bandwidth bounds on end-to-end traffic flows transported by a best-effort wormhole NoC fabric with no special hardware support for RT traffic. Our approach is inspired by the work by Lee et al. [10] for traditional multiprocessor networks, which we extend in several directions.

We propose two different methods for characterizing worst-case packet injection. The first method, RTB-HB (Real-Time Bound for High-Bandwidth traffic), is used for NoCs supporting application workloads where the injected flows have high demands of average bandwidth and require a guaranteed worsttraffic minimum bandwidth $(m B W)$ and maximum upper bound latency $(U B)$. In this case we do not assume any restriction on traffic injection rate: a flow can send packets whenever the network has buffer capacity to accept them. The second method considers applications with latency-critical flows which require low and guaranteed UB values, but have moderate bandwidth requirements and do not send packets at intervals shorter than a minimum permitted interval - which obviously implies a maximum bandwidth $(M B W)$ limitation. This method, called RTB-LL (Real-Time Bound for Low-Latency traffic) requires a very simple traffic regulation at network injection points. RTB$\mathrm{LL}$ is a significant improvement to the WCFC bound proposed in [10], while RBT-HB is completely new. Table 1 summarizes a cross-comparison of RTB-HB, RTB-LL and WCFC methods.

\footnotetext{
${ }^{1}$ This work has been financially supported partly by EU ARTIST-DESIGN and Predator projects and also partly by Iran Telecommunication Research Center (ITRC), an affiliation of the Ministry of Communications and Information Technology.
} 
Table 1: Upper bound delay and bandwidth comparison in different methods

\begin{tabular}{|c||c|c|}
\hline Methods & WCFC (W) & RTB-LL (LL) \\
\hline \hline RTB-HB & $U B_{H B} \leq U B_{W}$ & $U B_{H B} \geq U B_{L L}$ \\
(HB) & $m B W_{H B} \geq M B W_{W}$ & $m B W_{H B} \leq M B W_{L L}$ \\
\hline RTB-LL & $U B_{L L} \leq U B_{W}$ & \\
(LL) & $M B W_{L L} \geq M B W_{W}$ & \\
\cline { 1 - 2 } & &
\end{tabular}

The remainder of the paper is organized as follows. Section 2 summarizes related work. Section 3 gives definitions and basic concepts. Section 4 describes methods RTB-HB and RTB-LL. Section 5 focuses on experimental results and quantitative comparisons. Section 6 describes the time complexity of the methods. Finally, section 7 concludes the paper.

\section{RELATED WORK}

The body of knowledge on macro-scale RT networks is extensive and an overview of the state-of-the-art is beyond the scope of this work. The interested reader is referred to [11 20]. Here we focus on RT-NoCs, which have often been called QoS-NoCs.

QoS is an important issue for many application domains, such as multimedia, aerospace and military applications. Many of these applications have one or more traffic flows that have real time requirements and need hard QoS guarantees. Some NoC architectures provide hard QoS support by using special hardware mechanisms. In [9], Goossens et. al present the Æthereal NoC, which combines guaranteed services with best effort services to guarantee QoS in NoCs. The MARS [21] architecture uses TDMA (Time division multiplexing) mechanism to provide real-time guarantees on packet switched networks. In Shi et. al [7], a priority-based wormhole switching method for scheduling of RT flows is presented. In [8], Paukovits et. al propose the concept of a predictable TimeTriggered Network-on-Chip (TTNoC) that realizes QoS based communication services. Many other works have been published with variations and improvements over these basic ideas [22-33]. However, most NoC architectures are best-effort [34] and do not have special hardware mechanisms to guarantee QoS. Today, there is no available method that can calculate worst-case bandwidth and delay values of a general best-effort NoC design. Our work addresses this open issue.

\section{NETWORK MODEL}

A router model is essential to characterize network latency and bandwidth. We consider the very general reference architecture shown in Fig. 1 where a crossbar handles the connections among input and output channels. For maximum generality, we consider buffering at input and (optionally) output ports. We assume round-robin arbitration in the switches, a commonly used arbitration scheme in many NoCs. Virtual channels can be supported by the proposed methods. Links, which can be pipelined to maximize the operating frequency, connect the output ports to the input ports of adjacent switches. Table 2 summarizes the parameters that we will use in the following to describe the network. For the sake of simplicity, we use a single parameter Freq for the operating frequency of all cores and a single FlitWidth as the data width of the NoC links.
Table 2: Network model parameters and symbols

\begin{tabular}{|c|l|}
\hline Parameter & Description \\
\hline Freq & Clock frequency of the system \\
\hline$a$ & Number of pipeline stages (registers) used to segment NoC links \\
\hline$b_{1}$ & Depth of switch input buffers \\
\hline$b_{2}$ & Number of pipeline stages of the switch crossbar ( 0 if combinational) \\
\hline$b_{3}$ & Depth of switch output buffers ( 0 if none) \\
\hline$b$ & $:=b_{1}+b_{2}+b_{3}$ \\
\hline$B_{d}$ & Buffer depth parameter, $:=a+b_{1}+b_{2}+b_{3}=a+b$ \\
\hline$t s_{1}$ & Latency overhead for injecting a packet into the network \\
\hline$t s_{2}$ & Latency overhead for ejecting a packet at the destination \\
\hline$F l i t W i d t h$ & Width of NoC links, in bytes \\
\hline$S W_{j}$ & $j$-th switch of the network \\
\hline
\end{tabular}

The buffer depth $\left(B_{d}\right)$ parameter will be used frequently in the paper. As can be seen in Fig. 1, $B_{d}$ is the summation of the number of registers or buffers from the arbitration point (at the entry of the crossbar) of switch $j$ to the arbitration point of switch $j+1 . b_{1}$ is the depth of the input buffer (we assume at least one register), $b_{2}$ is the number of registers in crossbar switch (if any), $b_{3}$ is the depth of the output buffer (if any), and $a$ is the number of registers (if any) along a link to compensate the propagation delay of the wires. It is important to note that $b_{2}$ and $a$ represent latencies that packets will face even in the absence of congestion, while $b_{1}$ and $b_{3}$ become most relevant in case of blocking, when buffers fill up; in the absence of congestion, input and output buffers can be traversed in a single cycle instead. Blocking always happens because of arbitration conflicts, either directly, when in front of a switch crossbar, or indirectly, due to full buffers ahead. For simplicity, throughout the paper, we consider the buffering between two adjacent switches to be lumped, so we mention 'output buffer of switch $j$ ' and 'input buffer of switch $j+1$ ' equivalently, referring to the same number of intermediate registers between the arbitration points of switches $j$ and $j+1$, i.e. to $B_{d}$. Please also note that the switches along a path are indexed $j=1 \ldots m$, but $j=0$ can conveniently be seen as a virtual switch inside the source node to model source conflicts (i.e. sending more than one flow from a source node).

The parameters $t s_{1}$ and $t s_{2}$ model the setup time at NoC sources and destinations to inject and eject packets. Of course, to be able to use finite parameters, we assume that the receiving nodes are able to accept incoming data at the required rates.

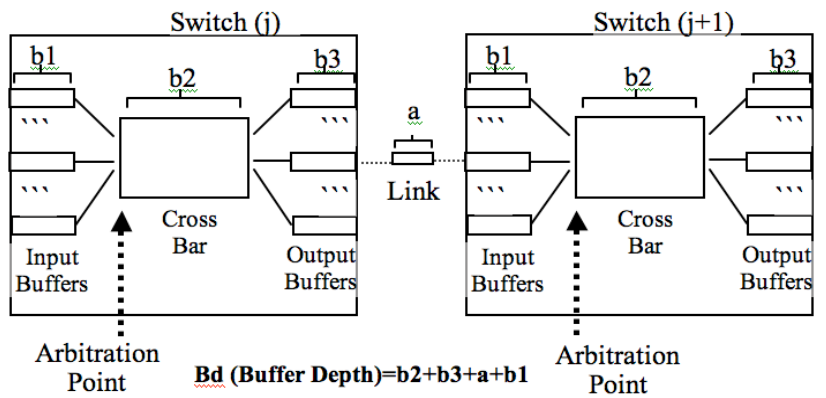

Figure 1: Switch model and parameters

\section{NOC TRAVERSAL DELAY ANALYSIS}

Table 3 lists the parameters we define to describe traffic flows across the network, while Table 4 summarizes the parameters that we identify to model the performance of such flows. Most 
notably, $U B_{i}$ represents the upper-bound delay for network traversal by a packet of flow $F_{i}$, and is a key figure for the interconnect designer. We try to use a notation as close as possible to that used in [10] for ease of comparison.

We first present a method called RTB-HB (Real Time Bound for High-Bandwidth traffic), which calculates $U B_{i}$ in a completely worst-case traffic situation. Crucially, this includes the possibility for other system cores to inject unregulated bandwidth, i.e. any amount of traffic at irregular intervals. This is a key property for real-world interconnect analysis methods, as most available IP cores operate on an unregulatedinjection basis. In order to calculate $U B_{i}$ in such a case, we consider all the intermediate buffers along the route full, and we assume arbitration losses at all switches against all other contending flows. Since switches are assumed to feature roundrobin arbitration, even though we assume the current flow to be serviced last, the maximum delay is bounded, i.e. starvation cannot occur. Therefore, the packets sent by the flow source $S_{i}$ are eventually delivered. RTB-HB calculates the interval $M I_{i}$, i.e. the number of cycles after which the output buffer of $S_{i}$ is guaranteed to be free again for further injection. From this value, the worst-traffic minimum injectable bandwidth $\left(m B W_{i}\right)$ can also be easily derived. Please note that this analysis can be applied to most NoC architectures, without any specific QoS hardware or software provisioning. We then move on to the description of another method, called RTB-LL (Real Time Bound for Low-Latency traffic). In this scenario, we assume that traffic injection can be regulated, as possible in some application scenarios. Therefore, we also calculate a minimum permitted interval $\left(m I_{i}\right)$ between two consecutive packets from the same source, which can be translated into a maximum permitted bandwidth $\left(M B W_{i}\right)$. This approach is similar to previously published method [10] (Real-time wormhole channel feasibility checking or WCFC, which will be briefly described later), but delivers substantially better results in terms of bound tightness. For proper operation, the system must then respect $M B W_{i}$ bounds at runtime. Compared to method RTB-HB, in the calculations for method RTB-LL, it is possible to consider parameters $b 1$ and $b 3$ to represent the buffers delay $(b 1=1$ and $b 3=1$ or 0 , when there is no output buffer), instead of the exact buffer size.

\subsection{The Proposed Delay Model RTB-HB}

The goal here is the calculation of the parameters $U B_{i}$ (worstcase latency to traverse the network) and $M I_{i}$ (maximum worstcase interval). In this paper, due to lack of space, we only show the case where $B_{d}<=\mathrm{L}$. For a $B_{d}>\mathrm{L}$, we can introduce dummy switches for each port, each with $B_{d}<=\mathrm{L}$ and use the same analysis below. Let us first consider the case $B_{d}=L$. To grasp intuitively the analysis, please observe that $B_{d}=L$ means, in concrete terms, that a packet fills exactly the buffering resources between the arbitration points of two adjacent switches. Considering as an example a network completely full of traffic (an unrealistic scenario just for visualization purposes), and $B_{d}=L$ globally, the network operates by shuffling packets around in lockstep: all switches re-arbitrate simultaneously every $L$ cycles, and packets trail each other, filling up buffers as soon as they free up.
Table 3: Traffic model parameters

\begin{tabular}{|l|l|}
\hline Parameter & Description \\
\hline$F_{i}$ & $i$-th traffic flow in the network \\
\hline$L_{i}$ & Length of the packets of $F_{i}$, in flits \\
\hline$S_{i}$ & Source node of $F_{i}$ \\
\hline$D_{i}$ & Destination node of $F_{i}$ \\
\hline$P_{i}$ & Packet of $F_{i}$ \\
\hline$h_{i}$ & Number of switches (hops) along the path of $\mathrm{F}_{\mathrm{i}}$ \\
\hline
\end{tabular}

Table 4: Performance model parameters

\begin{tabular}{|c|c|}
\hline Parameter & Description \\
\hline$U B_{i}$ & Upper bound delay for a packet $P_{i}$ of $F_{i}$ to traverse the NoC \\
\hline$M I_{i}$ & $\begin{array}{l}\text { Maximum interval until the ability to inject a new packet of } F_{i} \text {, } \\
\text { used in method RTB-HB }\end{array}$ \\
\hline$m I_{i}$ & $\begin{array}{l}\text { Minimum permitted interval between two consecutive packets of } \\
F_{i} \text {, used in methods WCFC and RTB-LL }\end{array}$ \\
\hline$m B W_{i}$ & $\begin{array}{l}\text { Worst-traffic minimum injectable bandwidth for } F_{i} \text {, used in } \\
\text { method RTB-HB and is equal to: } m B W_{i} \\
:=L^{*} \text { FlitWidth } / \text { MI }_{i}^{*} \text { Freq }\end{array}$ \\
\hline$M B W_{i}$ & $\begin{array}{l}\text { Maximum permitted bandwidth for } F_{i} \text {, used in methods WCFC } \\
\text { and RTB-LL and is equal to: } M B W_{i}:=L^{*} \text { FlitWidth/mI } I_{i}^{*} \text { Freq }\end{array}$ \\
\hline$u_{i}^{j}$ & $\begin{array}{l}\text { The time needed for } P_{i} \text { to go from the input buffer of } S W_{j}(j>0) \\
\text { or from generating process in } S_{i}(j=0) \text { to the input buffer of } S W_{j+1} \\
\left(0 \leq j \leq h_{i}-1\right) \text { or } D j\left(j=h_{i}\right)\end{array}$ \\
\hline$U_{i}^{j}$ & $\begin{array}{l}\text { The time needed for } P_{i} \text { to go from the output buffer of } S W_{j}(j>0) \\
\text { or from the output buffer of } S_{i}(j=0) \text { to the output buffer of } \\
S W_{j+1} \text { this parameter is used for convenience and, mathematically } \\
\text { it can be written based on } u_{i}^{j} \text {, as } U_{i}^{j}=u_{i}^{j+1}, j \geq 0\end{array}$ \\
\hline$z_{0}(i, 0)$ & Number of flows contending with $F_{i}$ at $S_{i}$ \\
\hline$z_{c}(i, j)$ & Number of flows contending with $F_{i}$ at $S W_{j}$ at output channel $c$ \\
\hline$I(x)$ & Index of the $x$-th flow contending with $F_{i}$ at $S_{i}\left(\right.$ or $\left.S W_{j}\right)$ \\
\hline
\end{tabular}

More formally, when $P_{i}$ is generated in $S_{i}$, we consider all intermediate buffers along its route as full of packets from different flows. In the worst case, for $P_{i}$ to reach its destination, all these packets must leave their buffers. Focusing e.g. on hop $j, P_{i}$ may have arbitration conflicts with a number $z_{c}(i, j)$ of other flows contending for the output channel $c$, for example flows $F_{q}$ and $F_{r}$. Since round robin arbitration is assumed, it is enough to consider all contending flows to send a packet before $P_{i}$ to guarantee that the analysis is worst-case. The order in which contending flows obtain the arbitration is not important for the calculation of the delay of $P_{i}$. So $P_{q}$ should make a one-hop forward progress. While $P_{q}$ frees the buffers at hop $j$ flit by flit, the flits from $P_{r}$ will smoothly replace the free buffer spaces. Eventually $P_{i}$ also goes through. Section 4.3 presents a simple example to visualize this. The parameter $u_{i}{ }^{0}$ represents the time needed for $P_{i}$ to be ejected from $S_{i}$ and be placed in the output buffer of $S_{i}$ (or input buffer of the first switch of $F_{i}$ ). $u_{i}^{j}$ then represents the time needed for $P_{i}$ to go from the input buffer of $S W_{j}$ to the input buffer of $S W_{j+1}$, except for the last switch. At the last switch $P_{i}$ is ejected, so it is instead the time needed to get into the input buffer of destination $D_{i}$. To calculate $U B_{i}$, as shown in Eq. 1, all these contributions must be added up, plus the fixed overhead for packet creation and ejection.

$$
U B_{i}=t s_{1}+t s_{2}+\sum_{j} u_{i}^{j} \quad \text { with } j=0 \ldots h_{i}
$$

The time needed for $S_{i}$ to inject the next packet is the time to create such a packet, plus the time needed for this packet to move on to the input buffer of the first switch:

$$
M I_{i}=t s_{1}+u_{i}^{0}
$$

To be consistent with the notations from [10] and for convenience, we introduce the uppercase $U_{i}^{j}$ symbol, which models the hop delay from output buffer to output buffer, instead of from input buffer to input buffer. 
Let us consider a packet of flow $F_{i}$ initiated at the source $S_{i}$. For this packet to reach the input buffer of the first switch, any already existing packet at that buffer has to leave, freeing the buffer. This existing packet could be a previous packet from the same flow $F_{i}$ or any of the contending flows at the output channel of the source. Thus, the worst-case time taken for any existing packet to leave the buffer is given by $\operatorname{MAX}_{x}\left(U_{i}^{0}\right.$ , $U_{I(x)}^{0}$ ), where $I(x)$ is the index of contending flows at the output channel, with $x=1 \ldots z_{0}(i, 0)$. Also, all the other contending flows of $F_{i}$ may have to send a packet before this flow. Thus, the total delay for a packet from $F_{i}$ to reach the input buffer of the first switch is given by:

$u_{i}^{0}=\operatorname{MAX}\left(U_{i}^{0}, U_{I(x)}^{0}\right)+\sum_{x} U_{I(x)}^{0}$, with $x=1 \ldots z_{0}(i, 0)$

For the subsequent hops, $u_{i}^{j}$ can be calculated similarly:

$$
\begin{aligned}
& u_{i}^{j}=\operatorname{MAX}\left(U_{i}^{j}, U_{I(x)}^{j}\right)+\sum_{x} U_{I(x)}^{j} \text { with } \\
& x=1 \ldots z_{c}(i, j), 1 \leq j \leq h_{i}
\end{aligned}
$$

Please note that, if there is no contention for the flow, then the above equation reduces to $u_{i}^{j}=U_{i}^{j}$. This is again akin to a packet moving in a pipeline fashion in the network.

In order to calculate the $U_{i}^{j}$ values, let us consider the packet from flow Fi moving from output buffer of the source to the output buffer of the first switch. For the packet to move, any existing packet from the output buffer of the first switch should move to the output buffer of the second switch. Similar to the above calculations, the maximum delay for this is given by $\operatorname{MAX}_{\mathrm{x}}\left(U_{i}^{j+1}, U^{j+1}{ }_{I}(x)\right)$. Please note the small difference from the $u_{i}^{j}$ calculations that, in this case, the values of $U_{i}^{j}$ at a switch $(j)$ depends on the values at the next switch $(j+1)$ on the path. The $U_{i}^{j}$ values can be obtained using the following equations:

$$
\begin{aligned}
& U_{i}^{j}=\operatorname{MAX}_{\mathrm{x}}\left(U_{i}^{j+1}, U^{j+1} I(x)\right)+\sum_{x} U^{j+1} I(x) \\
& \text { with } \quad x=1 . . z_{c}(i, j+1), 0 \leq j \leq h_{i}-1
\end{aligned}
$$

For the case of the last switch, from the output port, the packet can be ejected in $L_{i}$ cycles, with one flit of the packet ejected each cycle. Thus,

$$
U^{h i}{ }_{i}=L_{i}, U^{h_{(X)}} I(x)=L_{I(x)}
$$

Based on Eq. 3 and 4, now the problem of finding $U B_{i}$ and $M I_{i}$ (Eq. 1 and 2) is mapped onto a summation of $U_{i}^{j}$ values, which can be solved by Eq. 5. Please note that we assume the destination have enough buffers to eject the packets at the rate at which the network delivers them. By applying the above formulae recursively, we can obtain the worst-case delay (UB) and injection rate (MI) for the different flows. The same set of formulae also apply to the case where $B_{d}<L$. It is intuitive that the queuing effects are similar for this case as that of $B_{d}=L$. The methods can be easily extended to support virtual channels and different message lengths, so in the examples throughout the paper, we have used $L_{i}$ instead of $L$. But due to lack of space, we omit these extensions in this paper.

\subsection{The Proposed Delay Model RTB-LL}

We present a substantial improvement to the previously published method WCFC [10]. WCFC also calculates upper bound propagation delays and permitted injection intervals for flows in wormhole networks. It considers the arbitration contention that packets will face and, recursively, the delay incurred by other packets sharing some part of their route due to these blockings. With the same notation as above, WCFC proposes [10] the following formulae for calculating $U B_{i}$ and $m I_{i}$ :

$$
\begin{aligned}
& U B_{i}=t s_{1}+t s_{2}+L_{i}+\left(h_{i}+1\right) a+\sum_{j} u_{i}^{j} \quad \text { with } j=0 \ldots h_{i} \\
& m I_{i}=t s_{I}+L_{i}+\sum_{j} u_{i}^{j}-h_{i} b \quad \text { with } j=0 \ldots h_{i}
\end{aligned}
$$

where

$$
u_{i}^{j}=b+\sum_{x} U_{I i(x)}^{j} \quad \text { with } x=1 \ldots z_{c}(i, j)
$$

In the WCFC method, the calculations are based on the assumption that each flow injects packets spaced by at least a minimum permitted interval. For applications that can support such an assumption, we present a method that gives significant improvement over the WCFC method, which we call RTB-LL. To improve upon WCFC, a new concept of overlapping flows is introduced. If two or more different flows contend for the same output port at a switch, and they also share the same input port, we call such flows overlapping at the switch. This notion allows us to significantly optimize the bound tightness.

When $F_{i}$ contends with multiple overlapping flows at a switch, it is possible to locally coalesce all such overlapping flows into a single one. This is because the arbitration cannot be lost to multiple of those flows, as they cannot physically produce a contending packet simultaneously given that they enter the switch through the same input port. If there exist e.g. two overlapping contending flows at hop $j$ having delay parameters $U_{i 1}^{j}$ and $U_{i 2}^{j}$, then it is possible to consider $\operatorname{MAX}\left(U_{i 1}^{j}, U_{i 2}^{j}\right)$ as their representative delay, instead of $U_{i 1}^{j}+U_{i 2}^{j}$, for calculating the parameters for $F_{i}$, and so the main contending flows $\left(i_{l}\right.$ and $i_{2}$ ) are ignored in the calculations. When $F_{i}$ overlaps with other flows, in the calculations for $F_{i}$ the other contending flows should be ignored. By applying these optimizations, we have noticed a significant improvement in bound tightness in RTB$\mathrm{LL}$, as will be seen in next section and as Table 1 summarizes.

\subsection{Delay Calculation Examples}

To describe in detail different aspects of the analytical methods RTB-HB, RTB-LL and WCFC for upper bound delay and interval calculation, we apply them step-by-step to an example NoC (shown in Fig. 3) and then compare them. There are 4 message flows from $S_{1}$ to $D_{1}, S_{2,3}$ to $D_{3}, S_{2,3}$ to $D_{2,4}$ and $S_{4}$ to $D_{2,4}$. The NoC contains four switches. For the sake of simplicity, we consider $B_{d}=L$ in this example.

\subsubsection{Method RTB-HB}

As an example, we study the time needed for a packet $P^{0}$ of flow $F_{l}$ to get through the network. In general, from Eq. 1:

$$
U B_{1}=t s_{1}+t s_{2}+\sum_{j} u_{1}^{j} \quad \text { with } j=0 \ldots 3
$$

To start, let us model the time $u_{1}^{0}$ needed to move from $S_{l}$ to input buffer of switch $S W_{l}$. We start from the most congested network possible, so there exists another packet $P^{l}$ of the same flow ahead, and this packet needs $U^{0}{ }_{1}$ to go from the output buffer of the source (remember that source nodes are tagged with superscript 0 ) to the output buffer of $S W_{1}$, so $u^{0}{ }_{l}=U^{0}{ }_{1} . U^{0}{ }_{1}$ has to be calculated recursively based on the delays of the contending packets and of the packets ahead along the same route. 


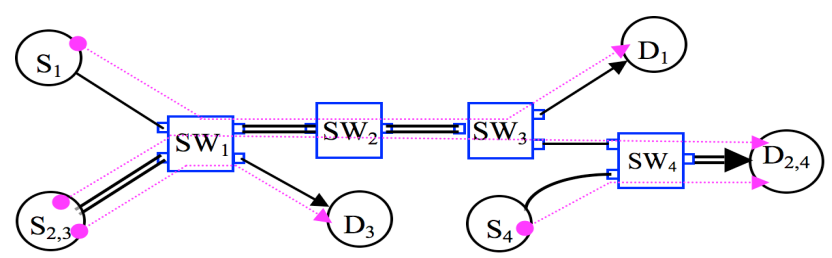

Figure 3. A simple example network

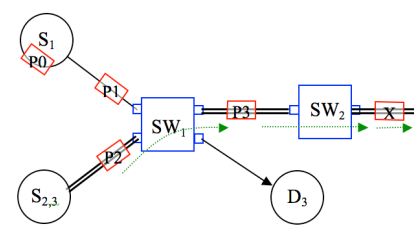

(a)

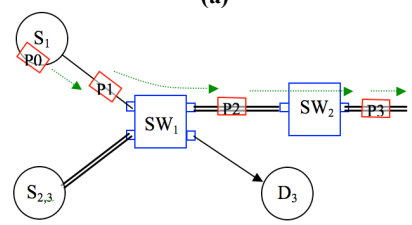

(b)

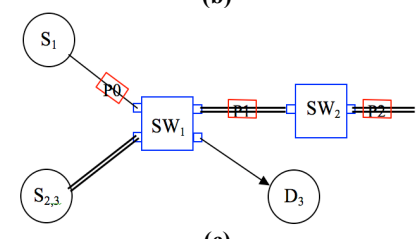

(c)

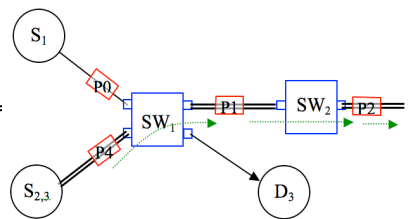

(d)

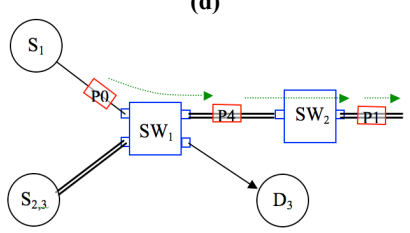

(e)

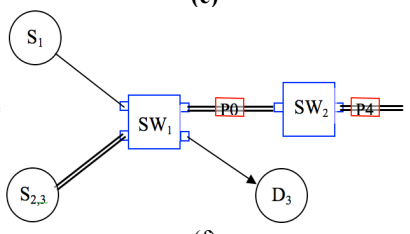

(f)

Figure 4: $(\mathrm{a}, \mathrm{b}, \mathrm{c})$ packet $\boldsymbol{P}^{0}$ goes from a process that generates it $\left(F_{1}\right.$ in $\left.S_{1}\right)$ to the input buffer of $S W_{1}$. (d, e, f) packet $P^{0}$ goes from input buffer of $S W_{1}$ to input buffer of $S W_{2}$

We observe that two factors contribute to its calculation: first, the possibility of losing arbitrations at $S W_{l}$; second, the fact that there may be no available buffer space at the output of $S W_{l}$ (due to arbitration losses ahead), which also effectively stalls packets at the input of $S W_{l}$. For what concerns the arbitration loss, it can be seen that flow $F_{1}$ contends with flow $F_{2}$ at the output of $S W_{l}$. Thus, a packet $P^{2}$ of $F_{2}$ currently in the input buffer of $S W_{1}$ could be arbitrated before $P^{l}$. For what concerns the output buffer full condition, in the worst case, there will be a packet $P^{3}$ in the output buffer of $S W_{1} . P^{3}$ could belong to either $F_{1}$ or $F_{2}$, in which cases, respectively, either $U^{l}{ }_{1}$ or $U^{l}{ }_{2}$ models the time for such packet to move ahead, from the output buffer of $S W_{l}$ to the output buffer of $S W_{2}$. $\operatorname{MAX}\left(U^{l}, U^{l}{ }_{1}\right)$ models the worst-case delay affecting our flow under study. During the time $\operatorname{MAX}\left(U^{l}{ }_{2}, U^{l}{ }_{l}\right)$, the packet $P^{3}$ moves on to the output buffer of $S W_{2}$, leaving the output buffer at $S W_{1}$ empty. However, in the worst case, an arbitration loss occurs to $P^{l}$, so it is packet $P^{2}$ which will smoothly replace $P^{3}$ (Fig. 4(a)). Before $P^{l}$ can move on by one hop, we must also consider the time for packet $P^{2}$ to go from the output buffer of $S W_{1}$ to the output buffer of $S W_{2}$ (Fig. 4(b)), which is $U^{l}{ }_{2}$. In summary: $\quad u_{1}^{0}=U^{0}{ }_{1}=\operatorname{MAX}\left(U^{l}{ }_{2}, U^{l}{ }_{l}\right)+U^{l}{ }_{2}$

Which traces back to Eq. 3. As mentioned above, this is the delay for $P^{l}$ to move one hop on, but equivalently is also the delay for $P^{0}$ to replace it in the previous location (Fig. 4(c)). Now, similarly, $P^{0}$ needs to move another hop on, from the input buffer of $S W_{1}$ to the input buffer of $S W_{2}$, with a delay which is defined as $u_{1}^{l}$.

It is possible to use the equation $u^{I}=U^{0}{ }_{1}$ based on the equality described in previous section, but for clarity we always describe $u_{i}^{j}$ based on $U_{i}^{j}$. As shown in Fig. 4(d, e, f), in the worst case, packet $P^{0}$ should wait for a packet $P^{4}$ of $F_{2}$. A packet $P^{l}$, again either from $F_{1}$ or $F_{2}$, should be considered at the output buffer of $S W_{l}$. So again, during the time $\operatorname{MAX}\left(U^{l}{ }_{2}, U^{l}{ }_{1}\right)$, while $P^{l}$ moves on to the output buffer of $S W_{2}, P^{4}$ will replace it. $P^{4}$ itself then takes $U_{2}^{I}$ to move on, allowing $P^{0}$ to eventually get to the input buffer of $S W_{2}$. Thus: $\quad u_{1}^{l}=\operatorname{MAX}\left(U^{l}{ }_{2}, U^{l}{ }_{1}\right)+U^{l}{ }_{2}$

In a similar manner $u_{l}^{2}$ can be calculated. Once $P^{0}$ is in the input buffer of $S W_{3}$, it is only one hop away from its destination and as there is no contending flow at the destination, the time that is needed to be ejected is equal to $L_{l}$. For uniformity of presentation we can write: $\quad u_{1}^{3}=U_{1}^{3}=L_{1}$

Now, the target metric $U B_{1}$ can be calculated recursively, as a function of a set of $U_{i}^{j}$ variables for the whole network starting from the last hop of each flow, where they assume a known value. The calculation of all intermediate values, e.g. $U^{l}{ }_{1}, U^{l}{ }_{2}, U^{2}{ }_{1}, U^{2}{ }_{2}$, $U^{3}{ }_{1}$, and of the relevant metrics $U B_{i}$ and $M I_{i}$, is shown in Fig. 5. Please note that intermediate values can be calculated once only and then stored, to speed up the recursion.

\begin{tabular}{|c|c|}
\hline 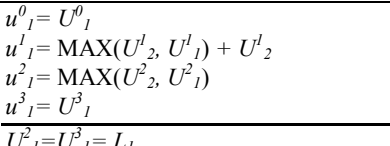 & $\begin{array}{l}u_{2}^{0}=\operatorname{MAX}\left(U^{0}{ }_{2}, U^{0}{ }_{3}\right)+U^{0}{ }_{3} \\
u_{2}^{I}=\operatorname{MAX}\left(U_{2}^{l}, U_{1}{ }_{1}\right)+U^{l}{ }_{1} \\
u_{2}^{2}=\operatorname{MAX}\left(U_{2}^{2}, U^{2}{ }_{1}\right) \\
u_{2}^{3}=U^{3}{ }_{2} \\
u_{2}^{4}=U_{4}^{4}+U^{4}{ }_{2}\end{array}$ \\
\hline $\begin{array}{l}U^{2}{ }_{2}=U^{3}{ }_{2}=U^{4}{ }_{4}+U^{4}{ }_{2}=L_{4}+L_{2} \\
U^{I}{ }_{1}=\operatorname{MAX}\left(U^{2}{ }_{1}, U^{2}{ }_{2}\right)=L_{2}+L_{4} \\
U^{I}{ }_{2}=\operatorname{MAX}\left(U^{2}{ }_{1}, U^{2}{ }_{2}\right)=L_{2}+L_{4}\end{array}$ & $\begin{array}{l}U_{2}^{0}=\operatorname{MAX}\left(U_{2}^{l}, U^{l}{ }_{l}\right)+U^{l}{ }_{l}= \\
2 L_{2}+2 L_{4} \\
U_{3}^{0}{ }_{3}=U^{l}{ }_{3}=L_{3}\end{array}$ \\
\hline$U^{0}{ }_{1}=\operatorname{MAX}\left(U^{l}{ }_{2}, U^{l}{ }_{1}\right)+U^{l}{ }_{2}=2 L_{2}+2 L_{4}$ & $U B_{2}=6 L_{2}+L_{3}+6 L_{4}$ \\
\hline$U B_{I}=L_{1}+5 L_{2}+5 L_{4}, M I_{I}=2 L_{2}+2 L_{4}$ & $M I_{2}=2 L_{2}+L_{3}+2 L_{4}$ \\
\hline $\begin{array}{l}u_{3}^{0}=\operatorname{MAX}\left(U_{2}^{0}, U^{0}{ }_{3}\right)+U^{0}{ }_{2} \\
u_{3}^{I}=U_{3}{ }_{3}=L_{3}\end{array}$ & $\begin{array}{l}u_{4}^{0}=U^{0}{ }_{4} \\
u_{4}^{4}=U^{4}{ }_{2}+U_{4}^{4}\end{array}$ \\
\hline$U B_{3}=4 L_{2}+L_{3}+4 L_{4}$ & $U_{4}^{0}=U_{2}^{4}+U_{4}^{4}=u_{4}^{4}$ \\
\hline$M I_{3}=4 L_{2}+4 L_{4}$ & $U B_{4}=2 L_{2}+2 L_{4}, M I_{4}=L_{2}+L_{4}$ \\
\hline
\end{tabular}

Figure 5: The complete calculation of $U B_{i}$ and $M I_{i}$ for the example NoC of Fig. 3 in method RTB-HB

When considering the source $S_{2,3}$, it can be noticed that two flows $F_{2}$ and $F_{3}$ can originate from it, therefore source conflicts may happen. As Fig. 5 shows, analyzing e.g. the flow $F_{2}$, in this case $u_{2}^{0}$ (the time to transfer of a packet of $F_{2}$ into the input buffer of the first switch) should include a delay $\operatorname{MAX}\left(U^{0}{ }_{2}, U^{0}{ }_{3}\right)$, which accounts for a packet of either $F_{2}$ or $F_{3}$ to move away from the input of $S W_{1}$ towards the input of $S W_{2}$ (during which time we must assume, in the worst case, that it is a packet of $F_{3}$ which replaces it), and then again the time $U_{3}^{0}$ for this latter packet to also move on, and finally letting a packet from $F_{2}$ in. The calculation for $u_{3}^{0}$ is the same.

\subsubsection{Methods RTB-LL and WCFC}

Fig. 6 and Fig. 7 show the calculated $U B_{i}$ and $m I_{i}$ values for both WCFC and RTB-LL methods for the same example of Fig. 3. Since flows $F_{1}$ and $F_{2}$ are overlapping at $S W_{2}$, our proposed RTBLL improves the bound tightness compared to WCFC. Considering for the sake of exemplification a NoC variant as in Fig. 8, with another contending flow at $S W_{2}$, then in RTB-LL the delay $u_{3}{ }_{3}$ of $F_{3}$ at $S W_{2}$ can be modeled as $b+\operatorname{MAX}\left(U_{1}^{2}, U^{2}{ }_{2}\right)$ instead of the overly pessimistic value $b+U^{2}{ }_{1}+U^{2}{ }_{2}$ calculated by WCFC. 
Table 5: Network parameters for the study

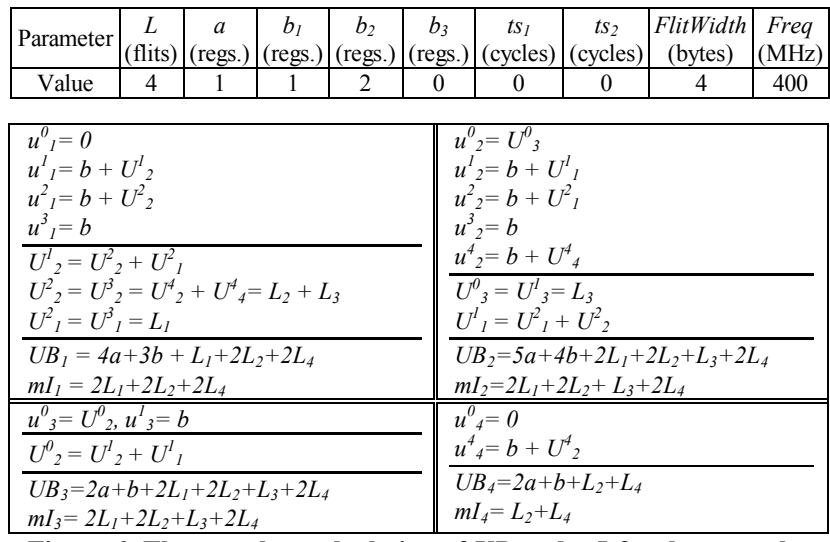

Figure 6: The complete calculation of $U B_{i}$ and $m I_{i}$ for the example NoC of Fig. 3 in method WCFC

\begin{tabular}{|c|c|}
\hline $\begin{array}{l}u_{1}^{0}=0 \\
u_{1}^{I}=b+U_{2}^{l} \\
u_{1}^{2}=b \\
u_{1}^{3}=b\end{array}$ & $\begin{array}{l}u_{2}^{0}=U_{3}^{0} \\
u_{2}^{I}=b+U^{l} \\
u_{2}^{2}=b \\
u_{2}^{3}=b\end{array}$ \\
\hline $\begin{array}{l}U_{2}^{l}=U^{2}{ }_{2}=U_{2}^{3}=U_{2}^{4}+U_{4}^{4}=L_{2}+L_{4} \\
U_{1}^{2}=U_{1}^{3}=L_{1}\end{array}$ & $\frac{u_{2}^{4}=b+U_{4}^{4}}{U_{3}^{0}=U_{3}^{l}=L_{3}}$ \\
\hline $\begin{array}{l}U B_{1}=4 a+3 b+L_{1}+L_{2}+L_{4} \\
m I_{1}=L_{1}+L_{2}+L_{4}\end{array}$ & $\begin{array}{l}U_{1}^{l}=U_{1}^{2} \\
U B_{2}=5 a+4 b+L_{1}+L_{2}+L_{3}+L_{4} \\
m I_{2}=L_{1}+L_{2}+L_{3}+L_{4}\end{array}$ \\
\hline$\frac{u_{3}^{0}=U_{2}^{0}, u_{3}^{I}=b}{U_{2}^{0}=U_{2}^{l}+U_{l}^{l}}$ & $\begin{array}{l}u_{4}^{0}=0 \\
u_{4}^{4}=b+U^{4}\end{array}$ \\
\hline $\begin{array}{l}U B_{3}=2 a+b+L_{1}+L_{2}+L_{3}+L_{4} \\
m I_{3}=L_{1}+L_{2}+L_{3}+L_{4}\end{array}$ & $\begin{array}{l}U B_{4}=2 a+b+L_{2}+L_{4} \\
m I_{4}=L_{2}+L_{4}\end{array}$ \\
\hline
\end{tabular}

Figure 7: The complete calculation of $U B_{i}$ and $m I_{i}$ for the example

NoC of Fig. 3 in method RTB-LL

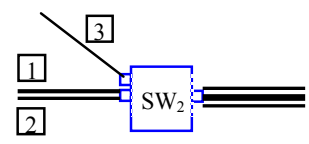

Figure 8: NoC variant where flow $F_{3}$ contends with two overlapping flows $F_{1}$ and $F_{2}$ at $S W_{2}$

\section{STUDIES ON APPLICATIONS}

The proposed methods RTB-HB and RTB-LL can be used to analyze the scheduling of traffic flows in real-world applications. In this section we present studies on five multimedia and RT applications, comparing RTB-HB and RTB-LL to the WCFC baseline, under the parameters of Table 5. In these applications, we assume that $\mathrm{NoC}$ topologies are predefined based on application communication needs, but without any feedback from the proposed algorithms to customize the network structure for better upper bound delay and interval time results. This is a possible extension for future work. In particular, for many applications it is possible to identify a small subset of few flows as critical, and then to optimize the NoC based on feedback loops from RTB-HB and RTB-LL to improve the performance of such critical flows. It is possible to do this without dedicated hardware support or any priority scheme.

\subsection{Case Study: A Multimedia Application}

In this section, we compare the results of applying RTB-HB, RTB-LL and WCFC to D26-media, a real-time multimedia application with 67 communications flows, some of which critical, shown in Fig. 9. The application is mapped onto two NoC topologies, one with 5 "fat" (high-radix) switches (shown in Fig. 10) and the other one with 20 "thin" (low-radix) switches. Fig. 11 presents the results of the study in terms of latency, interval and bandwidth for the whole set of flows. Fig. 11(a, b) compare the worst-case NoC traversal latency $U B_{i}$. The RTB-LL model always provides the tightest bounds. Compared to $\mathrm{WCFC}$, the largely improved tightness (more than $50 \%$ on average) is due to the analysis of overlapping flows, a novelty of this paper, but without any impact on the accuracy of the bounds, which are still under worst-case assumptions. RTB-HB naturally returns higher worst-case latency, due to the assumption that no hardware traffic injection regulation facilities are available. In fact, due to the different calculation approach, the bounds are on average still $30 \%$ lower than in WCFC, despite the less restrictive assumptions. There are, however, a few flows for which WCFC predicts lower delays than RTB-HB, due to the regulated injection assumption. In a zero-load scenario (no contention at all), the minimum theoretical latency to traverse the 5 -switch $\mathrm{NoC}$ for flows spanning a single hop is 8 cycles $\left(a+b_{1}+b_{2}+b_{3}+L\right)$, while RTB-LL gives a minimum upper-bound of 17 cycles in worstcase contention. In general, for all methods, the delays calculated for the 20-switch topology are higher, as a result of longer paths (more hops) per flow, higher probability of contention, and especially for RTB-HB more in-flight packets. This suggests, as intuitively expected, that NoCs with fewer hops guarantee lower delay bounds.

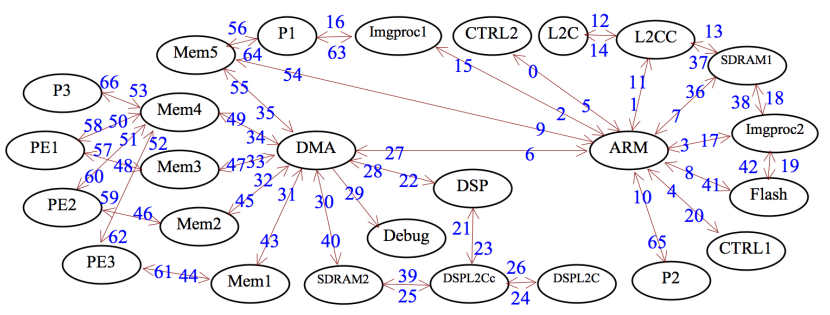

Figure 9: Communication graph for D26-media

Fig. 11(c, d) shows maximum and minimum injection intervals $\left(M I_{i}\right.$ and $\left.m I_{i}\right)$. Intuitively, if traversal delays are lower, new packets can be injected sooner, so $M I_{i}\left(m I_{i}\right)$ plots resemble $U B_{i}$ trends: flows with lower traversal latencies can be injected more frequently. Thus, the $m I_{i}$ intervals are always shorter in RTB-LL and the $M I_{i}$ intervals often shorter in RTB-HB when compared to $m I_{i}$ in WCFC. These intervals can be directly translated into minimum and maximum injectable bandwidths $\left(m B W_{i}, M B W_{i}\right)$ using the formulae in Table 3; results are shown in Fig. 11(e, f). Maximum injectable bandwidths $\left(M B W_{i}\right)$ are on average $35 \%$ higher according to RTB-LL compared to WCFC, and $25 \%$ higher according to the minimum bandwidth $\left(m B W_{i}\right)$ in RTB-HB. The maximum theoretical injectable bandwidth is $1600 \mathrm{MB} / \mathrm{s}$ (Freq* FlitWidth); according to RTB$\mathrm{LL}$, even under worst-case assumptions, some flows on the 5switch NoC are guaranteed injection rates of as much as 533 $\mathrm{MB} / \mathrm{s}$. In the 20-switch network, the higher contention likelihood affects injectable bandwidth negatively, but the use of more resources has a positive effect on many-hop flows, resulting overall in comparable injectable bandwidths. In 


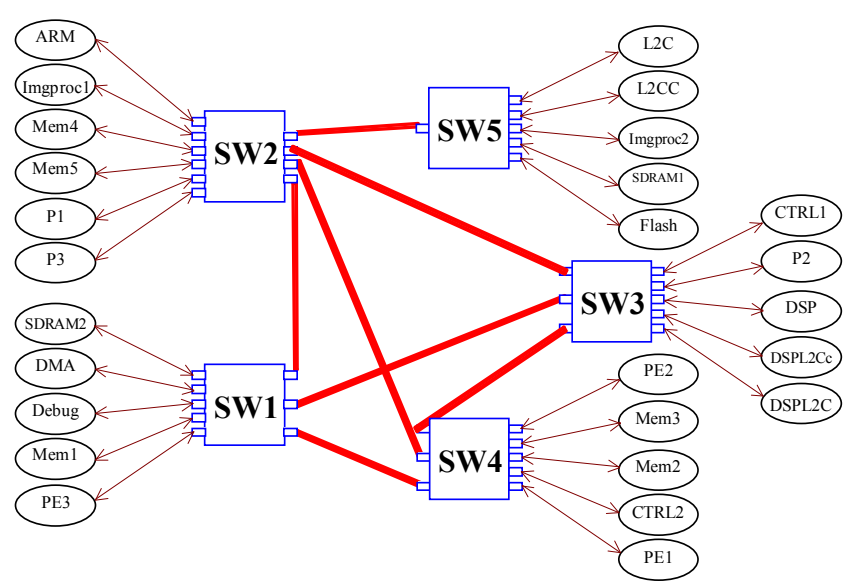

Figure 10 : D26-media application mapped on a 5-switch NoC

summary, NoCs with few hops exhibit clearly better upperbound traversal delays, but in terms of injectable bandwidths, the mapping of the flows (i.e. the contention patterns) and the amount of used resources play a decisive role in $\mathrm{NoC}$ performance.

\subsection{Suitability to Critical Flows}

Fig. 12 shows the average $U B_{i}$ traversal delay and the average $m B W_{i}$ injectable bandwidth for flows traversing $x$ hops of the 5switch NoC, considering the D26-media application and using RTB-HB. It can be seen that 1-hop flows exhibit reasonably low latencies and high bandwidths, suitable for critical traffic. Therefore, the proposed methodology has a clear applicability to industrial RT applications.

Table 6: Studied applications

\begin{tabular}{|c|c|c|c|c|}
\hline & Cores & $\begin{array}{c}\text { NoC } \\
\text { switches }\end{array}$ & $\begin{array}{c}\text { Clock frequency } \\
\text { (MHz) }\end{array}$ & $\begin{array}{c}\text { Traffic } \\
\text { flows }\end{array}$ \\
\hline D26-media & 26 & 5 & 400 & 67 \\
\hline Pipeline & 65 & 6 & 300 & 378 \\
\hline Bottleneck & 35 & 6 & 300 & 128 \\
\hline 36core-4 & 36 & 6 & 400 & 144 \\
\hline 36core-6 & 36 & 7 & 400 & 216 \\
\hline
\end{tabular}

\subsection{Comparison for Different Applications}

Fig. 13 shows the implementation results of the 3 different methods to the five applications listed in Table 6. 36core- 4 and 36 core- 6 are different mainly because in the former application each core handles 4 communication flows to as many other cores, while in the latter each core handles 6 such flows. Fig. 13 proves that, for all the applications, RTB-HB and RTB-LL provide tighter average bounds than the reference WCFC method. RTB-LL is strictly tighter than WCFC, while RTB$\mathrm{HB}$, although it provides much tighter bounds on average, can return higher bounds for specific flows since it does not rely on regulated traffic assumptions of any kind.

\section{COMPLEXITY OF THE METHODS}

To estimate the time complexity of the proposed algorithms, implemented for the proposed methods, we calculate the maximum number of operations that are required. As Eq. 1, Eq. 3 and Eq. 5 show, the only operations are additions and comparisons (for the MAX operator); so we may consider one cycle to execute each of these operations.

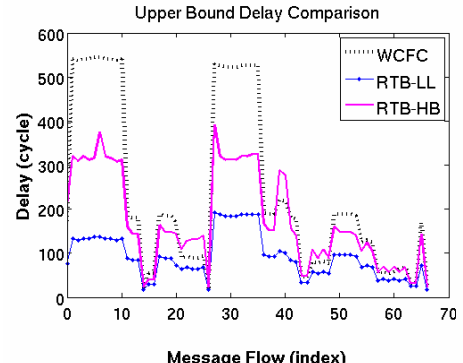

RTB-LL:min, avg, max:17,91,193 RTB-HB:min, avg, $\max : 24,174,392$ WCFC :min, avg, $\max : 17,237,545$ (a)

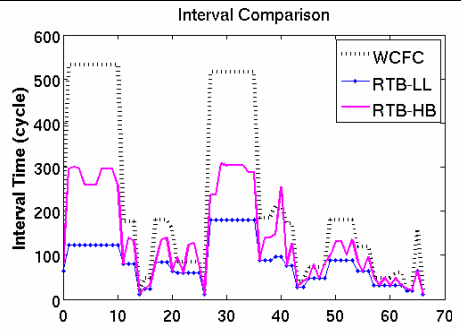

Message Flow (index)

RTB-LL:min, avg, $\max : 12,84,180$ RTB-HB:min, avg, $\max : 12,139,308$ WCFC :min, avg, $\max : 12,229,532$ (c)

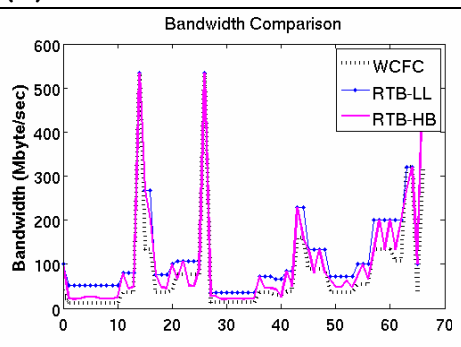

RTB-LL:min, avg, $\max : 36,124,533$ RTB-HB:min, avg, $\max : 21,101,533$ WCFC :min, avg, $\max : 12,81,533$

(e)

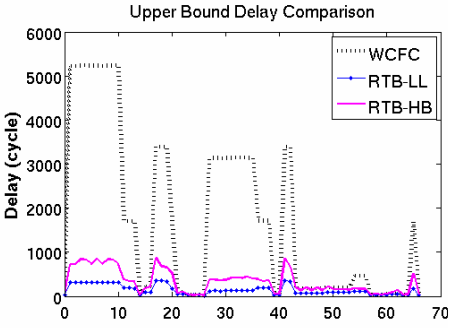

Message Flow (index)

RTB-LL:min, avg, $\max : 17,143,357$ RTB-HB: $m i n, a v g, \max : 24,329,868$ WCFC : min, avg, $\max : 17,1728,5233$ (b)

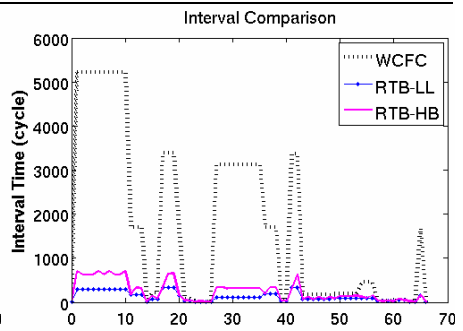

Message Flow (index)

RTB-LL:min, avg, $\max : 12,132,340$ RTB-HB:min, avg, max:12, 245, 708 WCFC:min, avg, $\max : 12,1717,5216$ (d)

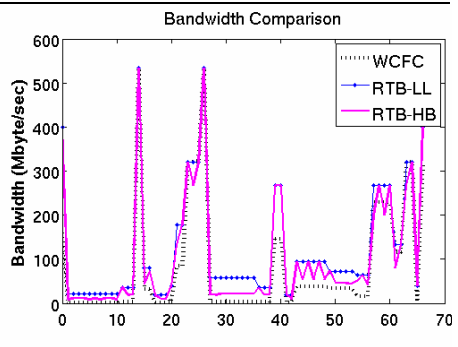

RTB-LL:min, avg, $\max : 19,121,533$ RTB-HB:min, avg, $\max : 9,103,533$ WCFC : min, avg, $\max : 1,79,533$

(f)

Figure 11: (a, c, e) $U B_{i}, M I_{i}$ and $m B W_{i}$ characterized with WCFC, RTBLL and RTB-HB for D26-media mapped onto a 5-switch NoC. (b, d, f) The same metrics mapping on 20 -switch NoC. Horizontal axes enumerate the communication flows.

We call $h$ the maximum number of switches in a flow, and $k$ the number of flows. We also pessimistically assume the maximum number of contending flows at a switch output to be $k$. For calculating one $U_{i}^{j}$ parameter, we need (Eq. 5) at most $k$ comparisons and $k$ additions, thus $2 k$ operations. The number of $U_{i}^{j}$ parameters to be calculated is $h k$, so the maximum number of operations is $2 h k^{2} . u_{i}^{i}$ parameters (except for $j=0$ ) can be derived from the equality $U_{i}^{j}=$ $u^{j+1}{ }_{i}$, so we only need to calculate the case of $u_{i}^{0}$ for all flows. In this case, one $u_{i}^{0}$ (Eq. 3) needs $2 k$ operations; for all $u_{i}^{0}$ parameters we need $2 k^{2}$ operations.

In RTB-HB the outcome are $k U B_{i}$ and $k M I_{i}$ values. For calculating one $U B_{i}$ value (Eq. 1 ) we need $h+1$ additions, so for all $k U B_{i}$ values we need $(h+1) k$, while in the case of $M I_{i}, k$ operations are needed. The total number of operations is the summation of all the above, or $2 h k^{2}+2 k^{2}+(h+1) k+k$. So the complexity of the algorithm is $O\left(h k^{2}\right)$. 


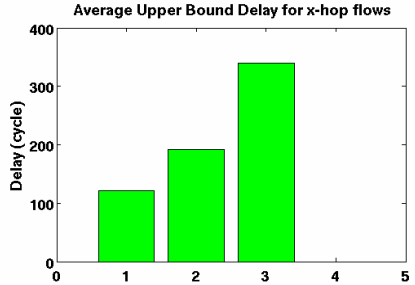

Number of hops (switches) per Flow

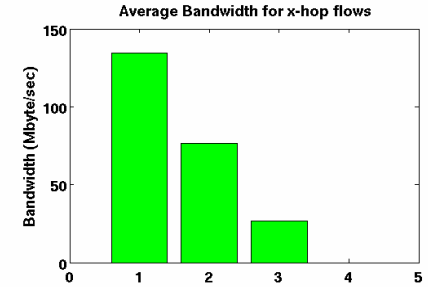

Number of hops (switches) per Flow

Figure 12. Average Upper Bound Delay (left) and Average Minimum Bandwidth (right) for $\mathrm{x}$-hop flows in D26-media for RTB-HB

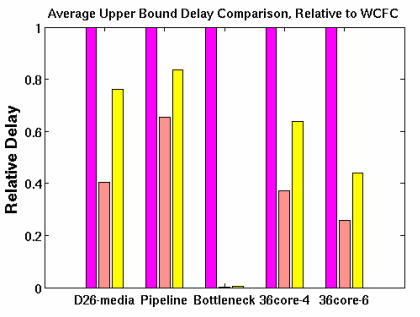

Application - WCFC/RTB-LLRTB-HB

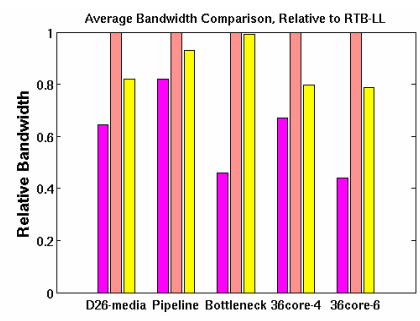

Application - WCFC/RTB-LL/RTB-HB
Figure 13. Comparisons: (left) average $U B_{i}$ (WCFC as reference), (right) average $M B W_{i}$ or $m B W_{i}$ (RTB-LL as reference)

For RTB-LL, using the same approach, we can show that the complexity of the algorithm for calculating $U B_{i}$ and $m I_{i}$ is again $O\left(h k^{2}\right)$. Thus, both algorithms have quadratic time complexity. In practice, the execution time for all our test applications is very small (few seconds on a standard PC) and the modeling of delay and bandwidth parameters does not pose significant runtime issues.

\section{CONCLUSION AND FUTURE WORKS}

We have proposed two different methods to characterize bandwidth and latency for NoC-based real-time SoCs, aiming at guaranteed QoS provisions. The choice of the most suitable method depends on the performance demands of the system and on whether dedicated hardware facilities can be supplied in the NoC. One method is aimed at applications demanding minimum latencies and requires injection regulation, while the other is suitable for applications where packet injection must be flexible to accommodate for higher average injected bandwidths and no hardware regulation is available. We proved that the proposed methods return the worst-case metrics in a much tighter way than existing approaches, rendering them quite applicable for real-world SoC applications. The major next step is to use the results of this work as an input to an iterative procedure to synthesize optimized NoCs whereby the QoS demands of critical traffic flows are met.

\section{REFERENCES}

[1] W. J. Dally and B. Towles, "Route packets, not wires: On-chip interconnection networks, "Proceedings of the 38th Design Automation Conference, 2001.

[2] L. Benini and G. D. Micheli, "Networks on Chips: A New SoC Paradigm, “ Computer, 35(1):70-78, 2002.

[3] P. Guerrier and A. Greiner, "A generic architecture for on-chip packet-switched interconnections," in Proceedings of Design, Automation and Test in Europe Conference ands Exhibition(DATE '00), pp. 250-256, Paris, France, March 2000.

[4] Z. Guz, I. Walter, E. Bolotin, I. Cidon, R. Ginosar, and A. Kolodny, "Network Delays and Link Capacities in application-Specific Wormhole NoCs, " VLSI Design, Volume 2007, Article ID 90941
[5] J. Henkel, W. Wolf, and S. Chakradhar, "On-chip networks: a scalable, communication-centric embedded system design paradigm," in Proceedings of the 17 th International Conference on VLSI Design (VLSID '04), vol. 17, pp. 845-851, Mumbai, India, January 2004.

[6] S. Furber and J. Bainbridge. "Future trends in SoC interconnect, "In VLSI Design, Automation and Test, pages 183-186, 2005.

[7] Z. Shi and A. Burns, "Real-Time Communication Analysis for On-Chip Networks with Wormhole Switching, " Second ACM/IEEE International Symposium on Networks-on-Chip, 2008

[8] C. Paukovits, H. Kopetz, "Concepts of Switching in the Time-Triggered Networkon-Chip," Proceedings of the 2008 14th IEEE International Conference on Embedded and Real-Time Computing Systems, pp.120-12.

[9] K. Goossens, J. Dielissen, and A. Radulescu, "The Æthereal network on chip: Concepts, architectures, and implementations," IEEE Design and Test of Computers, 22(5):414-421,2005.

[10] S. Lee, "Real-time wormhole channels,“ J. Parallel Distrib. Comput. 63 (2003) 299-311

[11] D. Kandlur, K. Shin, D. Ferrari , "Real-Time Communication in Multihop Networks,“'IEEE Trans. on Para. and Distributed Systems, vol. 5, no. 10, Oct. 1994.

[12] M. Zhang, J. Shi, T. Zhang, Y. Hu, "Hard Real-time Communication over Multihop Switched Ethernet, “ Int. Conf. on Networking, Architecture, and Storage, 2008

[13] S. Gopalakrishnan, S. Lui, and M. Caccamo, "Hard Real-Time Communication in Bus-Based Networks, " In Proc. 25th IEEE Int. Real-Time Systems Symp., 2004.

[14] A. Yiming, and T. Eisaka, "A Switched Ethernet Protocol for Hard Real-Time Embedded System Applications," In 19th Conf. on Advanced Information Networking \& Applications, March 2005, pp. 41-44.

[15] K. Watson and J. Jasperneite, "Determining end-to-end delays using network calculus," In Proc. 5th IFAC Int. Conf. on Fieldbus Systems and Their Applications (IFAC-FET2003), July 7-8, 2003, pp. 255-260.

[16] J. Chen, Z. Wang, and Y. Sun, "Real-time capability analysis for switch industrial Ethernet traffic priority-based," In Proc. of Int. Conf. on Control Applications, Glasgow, UK, Sep. 2002, pp. 525-529.

[17] J. Jaspernite, P. Neumann, M. Theis, and K. Watson, "Deterministic Real-Time Communication with Switched Ethernet," In Proc. of WFCS'02, Vasteras, Sweden.

[18] S. Lee; K. C. Lee, and H. H. Kim, "Maximum communication delay of a realtime industrial switched Ethernet with multiple switching hubs," In 30th Conf. of IEEE Industrial Electronics Society, 2004.

[19] J. Loeser and H. Haertig, "Low-latency hard real-time communication over switched Ethernet," In Proc. of ECRTS 2004.

[20] J. Kiszka, B. Wagner, Y. Zhang, J. Broenink," RTnet - A Flexible Hard RealTime Networking Framework," In: 10th IEEE International Conference on Emerging Technologies and Factory Automation, 2005.

[21] H. Kopetz, A. Damm, C. Koza, et al., "Distributed fault-tolerant real-time systems: the Mars approach," IEEE Micro, 1989, 9(1): 25-40.

[22] E. Bolotin et al., "QNoC: QoS architecture and design process for network on chip," Journal of Systems Architecture, vol. 50, no. 2-3, pp. 105-128, Feb. 2004.

[23] T. Bjerregaardand, J. Sparsoe, "Arouter architecturefor connection-oriented service guarantees in the MANGO clockless network-on-chip," in DATE, vol. 2, Mar. 2005, pp. 1226-1231.

[24] A. Bouhraoua and M. E. Elrabaa, "A high-throughput network-on-chip architecture for systems-on-chip interconnect," in Intl. Symposium on Soc, Nov. 2006. [25] F. Felicijan and S. Furber, "An asynchronous on-chip network router with qualityof-service (QoS) support," in SOCC, Sep. 2004, pp. 274-277.

[26] N. Kavaldjiev et al., "A virtual channel network-on-chip for GT and BE traffic," in ISVLSI, vol. 00, Mar. 2006.

[27] A. Leroy et al., "Spatial division multiplexing: a novel approach for guaranteed throughput on NoCs," in CODES+ISSS, 2005, pp. 81-86.

[28] A. Mello, L. Tedesco, N. Calazans, and F. Moraes, "Evaluation of current QoS mechanisms in network on chip," in Intl. Symposium on Soc, 2006, pp. 115-118.

[29] M. Millberg, R. T. E. Nilsson, and A. Jantsch, "Guaranteed bandwidth using looped containers in temporally disjoint networks within the Nostrum network on chip," in DATE, 2004, pp. 890-895.

[30] F. Mondinelli, M. Borgatti, and Z. Vajna, "A 0.13 um 1Gb/s/channel store-andforward network on-chip," in SOCC, Sep. 2004, pp. 141-142.

[31] R. Mullins, A. West, and S. Moore, "The design and implementation of a lowlatency on-chip network," in ASP-DAC, 2006.

[32] A. Radulescu et al., "An efficient on-chip NI offering guaranteed services, sharedmemory abstraction, and flexible network configuration," IEEE Trans. ComputerAided Design of Integrated Circuits and Systems, vol. 24, no. 1, pp. 4-17, Jan. 2005.

[33] E. Rijpkema et al., "Trade offs in the design of a router with both guaranteed and best-effort services for network on chip," IEE Proc. Computers and Digital Techniques, vol. 150, no. 5, pp. 294-302, 2003.

[34] E. Salminen, A. Kulmala, T. Hamalainen, "Survey of Network-on-Chip Proposals," www.ocpip.org, March 2008. 